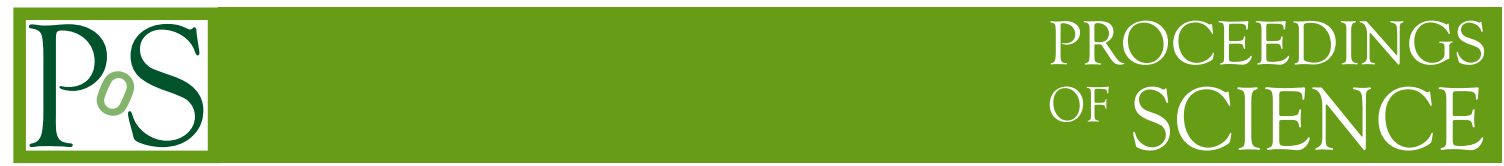

\title{
Progress on two-loop five-point Master Integrals
}

\section{Costas G. Papadopoulos ${ }^{* a}$ and Christopher Wever ${ }^{b, c}$}

a Institute of Nuclear and Particle Physics, NCSR Demokritos, Agia Paraskevi, 15310, Greece

${ }^{b}$ Institute for Theoretical Particle Physics (TTP), Karlsruhe Institute of Technology, D-76128

Karlsruhe, Germany

${ }^{c}$ Institut fr Kernphysik, KIT, 76344 Eggenstein-Leopoldshafen, Germany

E-mail: costas.papadopoulosecern.ch, christopher.wever@kit.edu

\begin{abstract}
A new approach to compute Feynman Integrals is presented. Based on it, we verify our previous results on planar five-point two-loop Master Integrals in the physical kinematical region. We also show how to obtain results for certain non-planar five-point two-loop Master Integrals in both Euclidean and physical regions.
\end{abstract}

Loops and Legs in Quantum Field Theory (LL2018)

29 April 2018 - 04 May 2018

St. Goar, Germany

\footnotetext{
* Speaker.
} 


\section{Introduction}

Almost seventy years from the time Feynman Integrals (FI) were first introduced [1-3] and more than forty-five years since the dimensional regularisation [4] set up the framework for an efficient use of loop integrals in computing scattering matrix elements, still the frontier of multiscale multi-loop integral calculations (maximal both in number of scales and number of loops) is determined by the planar five-point two-loop on-shell massless integrals [5,6], recently computed $^{1}$. On the other hand, in order to keep up with the increasing experimental accuracy as more data is collected at the LHC, more precise theoretical predictions and higher loop calculations are required [7].

Feynman graphs (FG) and the associated Feynman Integrals represent the building blocks of the perturbative expansion in Quantum Field Theory. Although to obtain results relevant for physics a lot of elements have to be combined, extending from parton-showers and resummation techniques to fixed-order calculations and parton-distribution functions, it is fair to say that any advancement seen so far in all these, is based on our knowledge of computing FI or closely related objects.

Scattering matrix elements at tree order (usually Leading Order, LO), where no loop-integrals appear, are best computed in the general case via off-shell recursive equations ${ }^{2}$, as well as through on-shell recurrence in the BCFW approach [11,12]. In a certain sense, a brute force computation of FG is not the most compact way to represent scattering matrix elements, a notable example being the Parke-Taylor amplitudes [13].

At the next-to-leading order (NLO), the reduction of one-loop amplitudes to a set of Master Integrals (MI), a minimal set of FI that form a basis, either relying on unitarity methods [14$16]$ or at the integrand level via the OPP method $[17,18]$, has drastically changed the way NLO calculations are preformed, resulting in many fully automated numerical tools (some reviews on the topic are $[19-21])^{3}$, making the NLO approximation the default precision for theoretical predictions at the LHC.

In the recent years, progress has been made also towards the extension of these reduction methods for two-loop amplitudes at the integral [22-31] $]^{4}$ as well as the integrand [32-37] level. Two-loop MI are defined using the integration by parts (IBP) identities [38-40], an indispensable tool beyond one loop. Contrary to the one-loop case, where MI have been known for a long time already [41], a complete library of MI at two-loops is still missing. At the moment this is one of the main obstacle to obtain a fully automated NNLO calculation framework similar to the one-loop one, that will satisfy the precision requirements at the LHC.

Many methods have been introduced in order to compute FI [42]. The overall most successful one, is based on expressing the FI in terms of an integral representation over Feynman parameters, involving the two well-known Symanzik polynomials $U$ and $F$ [43]. The introduction of the sector decomposition [44-48] method resulted in a powerful computational framework for the numerical evaluation of FI, see for instance SecDec [49]. An alternative is based on Mellin-Barnes repre-

\footnotetext{
${ }^{1}$ Complete results, including physical region kinematics, are published in [6].

${ }^{2}$ Known as Dyson-Schwinger [8,9] or Berends-Giele [10] equations.

${ }^{3}$ See also talks by Philipp Maierhöfer, Max Zoller, Fernando Febres Cordero and Malgorzata Worek

${ }^{4}$ Talk by Ben Page

${ }^{5}$ Talks by Simon Badger and William J. Torres Bobadilla
} 
sentation $[50,51]$, implemented in $[52]^{6}$. Nevertheless, the most successful method to calculate multi-scale multi-loop FI for the time being, is the differential equations (DE) approach [53-57], which has been used in the past two decades to calculate various MI at two-loops and beyond. Following the work of refs. [58-60], there has been a consensus, that the so-called multiple polylogarithms, more specifically the Goncharov Polylogarithms (GPs), form a functional basis for many MI, especially those with massless internal propagators. The so-called canonical form of DE, introduced by Henn [61], manifestly results in MI expressed in terms of GPs ${ }^{7}$. Nevertheless the reduction of a given DE to a canonical form is by no means fully understood, despite recent efforts [63-65]. Moreover it is well known that when for instance, enough internal masses are introduced, MI are not anymore expressible in terms of GPs, and in fact a new class of functions involving elliptic integrals is needed [66-70] $]^{8}$.

In this contribution we present a new approach to compute FI [71]. In Section 2, we present the basic idea of this method and give some examples. In Section 3, we use this new approach to obtain known results in an alternative way for two-loop five-point planar MI, and show how to obtain new results regarding the non-planar two-loop five-point MI. Finally in Section 4, we discuss the potential use and extension of it.

\section{Internal Reduction}

As in many areas of science, reducing a given problem to a simpler one, is considered as a very welcome virtue of any method. In this respect, a Feynman parameter is introduced, in order to appropriately combine internal propagators of a multi-loop FI. For instance two neighbour propagators with the same loop momentum are combined to a new one, as follows:

$$
\frac{1}{\cdots\left[\left(k+p_{1}\right)^{2}-m_{1}^{2}\right]\left[\left(k+p_{2}\right)^{2}-m_{2}^{2}\right] \cdots}=\int_{0}^{1} d x \frac{1}{\cdots\left[(k+q)^{2}-M^{2}\right]^{2} \cdots}
$$

with $q=x p_{1}+(1-x) p_{2}$ and $M^{2}=x m_{1}^{2}+(1-x) m_{2}^{2}-x(1-x)\left(p_{1}-p_{2}\right)^{2}$. By appropriately choosing the propagators to be combined, the resulting FI, which contains one internal line less, corresponds either to a simpler topology or to a FI with less external lines. In certain cases, it can be evaluated using IBP identities and the differential equations approach, in a much simpler way than the original one.

To illustrate the way Eq. (2.1) works, we start with a rather trivial example, shown graphically below

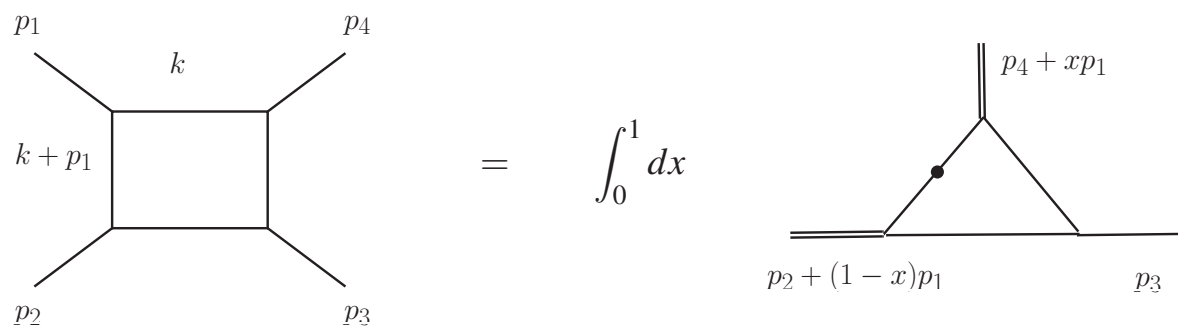

\footnotetext{
${ }^{6}$ See also https://mbtools.hepforge.org

${ }^{7}$ For an alternative method in the single scale case see also ref. [62]

${ }^{8}$ Talks by Claude Duhr, Lorenzo Tancredi and Stefan Weinzierl
} 
where all external particles momenta are incoming and light-like, $p_{i}^{2}=0, i=1, \ldots, 4$ and all internal particles are massless, both denoted graphically as single lines, whereas double lines correspond to external particles carrying momentum that satisfies $p^{2} \neq 0$ or to internal massive particles. The dotted line represents a squared propagator. As is well known, the triangle can be expressed in terms of bubble-integrals using IBP identities,

$$
\operatorname{Tr} \mathrm{i}=-\frac{2(d-3)}{S_{3}\left(S_{2}-S_{3}\right)} \mathrm{Bub}_{1}+\frac{2(d-3)}{S_{2}\left(S_{2}-S_{3}\right)} \mathrm{Bub}_{2}=\frac{2}{\varepsilon}\left[\frac{(-s)^{-1-\varepsilon}(1-x)^{-1-\varepsilon}}{s(1-x)-t x}-\frac{(-t)^{-1-\varepsilon} x^{-1-\varepsilon}}{s(1-x)-t x}\right]
$$

with $d=4-2 \varepsilon, S_{2}=\left(p_{2}+(1-x) p_{1}\right)^{2}=(1-x) s$ and $S_{3}=\left(p_{4}+x p_{1}\right)^{2}=x t, s=\left(p_{1}+p_{2}\right)^{2}$, $t=\left(p_{2}+p_{3}\right)^{2}$. So the box-integral in Eq. (2.2) is given by,

$$
\begin{aligned}
\text { Box } & =\int_{0}^{1} d x \text { Tri } \\
& =\frac{2}{\varepsilon^{2}} \frac{1}{s t}\left[(-s)^{-\varepsilon}{ }_{2} F_{1}\left(1,-\varepsilon ; 1-\varepsilon ; \frac{s+t}{t}\right)+(-t)^{-\varepsilon}{ }_{2} F_{1}\left(1,-\varepsilon ; 1-\varepsilon ; \frac{s+t}{s}\right)\right]
\end{aligned}
$$

a result known already for some time [72].

In general the IBP identities are not enough and we have to use the differential equations approach for the evaluation of the simpler FI. This is the case, for instance, in the following example

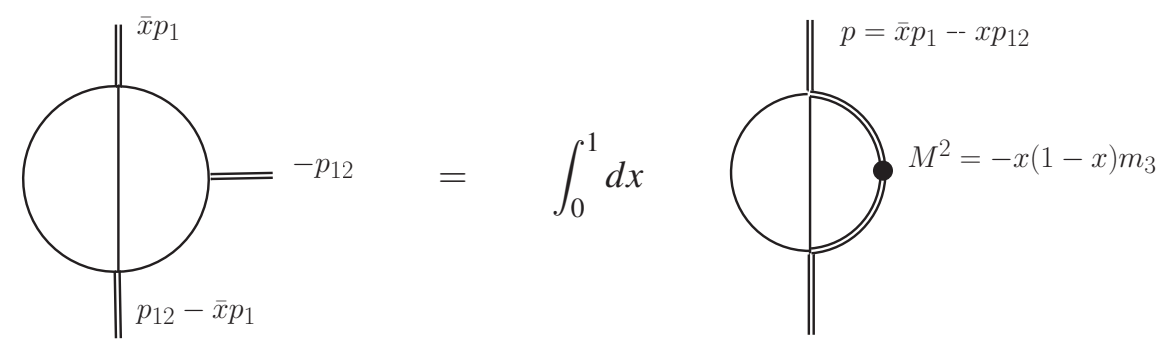

In Eq. (2.3), $p_{1}^{2}=m_{1}, p_{2}^{2}=0, p_{12}^{2}=m_{3}$ and the internal massive propagator is given by $P^{-1}=$ $\left(k_{2}-x p_{12}\right)^{2}+x(1-x) m_{3}$. Using the results of [73] we are re-deriving analytically the results of [74]. Details of the calculation can be found in [71]. Notice that, in this case, despite the fact that the Internal Reduction produces an $x$-dependent internal mass, solutions of the sunrise graph, obtained from DE, do allow for a straightforward analytic evaluation of the two-loop triangle, using Eq. (2.3).

\section{Using Internal Reduction for pentaboxes}

It is now a couple of years that we have calculated and provided full analytic results in terms of GPs for the pentabox planar family [6], shown here. 




The family consists of 74 MI, 3 of them in the highest 8-denominator sector. Using the simplified differential equations approach (SDE), we have managed to produce an $\varepsilon$-factorized form of the DE with respect to $\bar{x}$, with an alphabet of 19 letters (not depending on $\bar{x}$ ). Moreover, using the fact that the $\bar{x} \rightarrow 1$ limit is governed by the corresponding matrix-coefficient of the $1 /(1-\bar{x})$ term in the DE, we can also derive the result for all external particles carrying light-like momenta. Although we have been able to verify our results in the Euclidean region comparing with SecDec, a comparison with physical region kinematics was missing, due to the inadequacy of SecDec to produce results for the required kinematics.

Internal Reduction is offering a new possibility to compute these pentabox integrals. As shown in the figure below, we can relate the scalar pentabox MI with a planar double-box integral.
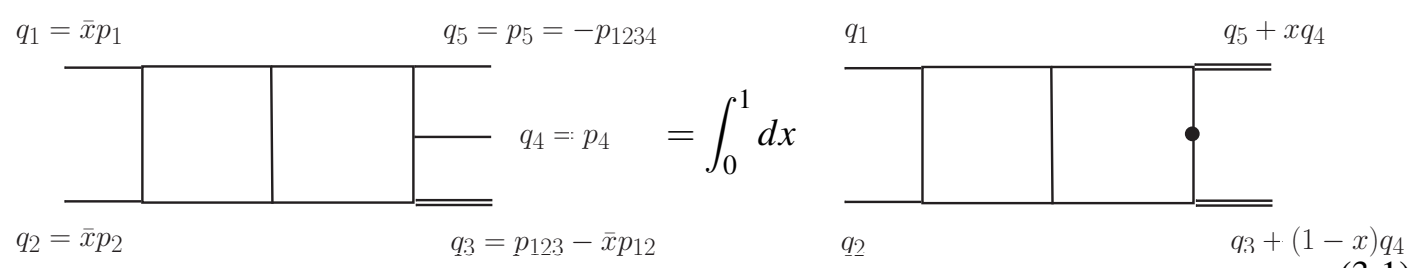

Taking advantage of the fact that we have in our disposal several results on planar double-box MI [75-77], we were able to numerically integrate Eq. (3.1) and compare with our published results, obtaining full agreement ${ }^{9}$. DE are indispensable to obtain this result, since we have to master the $x \rightarrow 1$ and $x \rightarrow 0$ limits of the double-box family MI in order to perform the integration in Eq. (3.1). Details of the calculation can be found in [71].

Finally, the scalar non-planar pentabox ${ }^{10}$ can be related, in the same manner, to the non-planar double-box family [76-78].

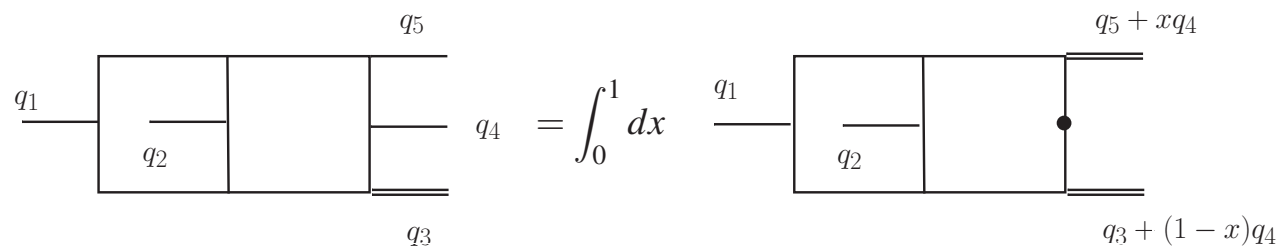

Details of the calculation and results for both physical and Euclidean kinematics and comparison with SecDec in Euclidean region can be found in [71].

\footnotetext{
${ }^{9}$ In the course of the Loops \& Legs 2018 Workshop, we have been informed by Nicola Adriano Lo Presti, in a private communication, that their results [5] are in agreement with ours in the physical region

${ }^{10}$ See also talk by Johannes Henn
} 


\section{Discussion and Outloook}

LHC high-luminosity phase will open a new frontier in precision physics. $\mathrm{N}^{3} \mathrm{LO}$ results for Higgs production are already available [79]. Eventually $\mathrm{N}^{5-n} \mathrm{LO}$ results for $2 \rightarrow n, n=1, \ldots, 3$ processes will be required at the end of the day ${ }^{11}$. It is therefore necessary to drastically simplify and to, as possible, fully automatise, the multi-loop calculations.

A new method, named as Internal Reduction, has been presented. We hope that it offers a realistic alternative in computing multi-scale multi-loop FI. It is based on a one-fold integral representation over a simpler FI, achieved by reducing one internal line. The method is making use of the knowledge of the DE through the SDE approach, for the simpler FI, although it can be used once DE are known in any form.

We are currently investigating the applicability of this method in several MI families, notably the missing non-planar 5-point two-loop ones. Numerical results are straightforwardly obtainable, once the DE for the reduced FI are known. Still, we believe that analytic results are possible, see [71] for details.

There are many possible extensions of the Internal Reduction method, the obvious one is to reduce more internal lines introducing more Feynman parameters. The efficacy of these ideas is currently under investigation.

\section{Acknowledgements}

I would like to thank Peter Marquard and Johannes Blümlein for their kind invitation and hospitality.

\section{References}

[1] R. P. Feynman, Space - time approach to quantum electrodynamics, Phys. Rev. 76 (1949) 769-789.

[2] F. J. Dyson, The Radiation theories of Tomonaga, Schwinger, and Feynman, Phys. Rev. 75 (1949) 486-502.

[3] F. J. Dyson, The S matrix in quantum electrodynamics, Phys. Rev. 75 (1949) 1736-1755.

[4] G. 't Hooft and M. J. G. Veltman, Regularization and Renormalization of Gauge Fields, Nucl. Phys. B44 (1972) 189-213.

[5] T. Gehrmann, J. M. Henn and N. A. Lo Presti, Analytic form of the two-loop planar five-gluon all-plus-helicity amplitude in QCD, Phys. Rev. Lett. 116 (2016) 062001, [1511.054 09].

[6] C. G. Papadopoulos, D. Tommasini and C. Wever, The Pentabox Master Integrals with the Simplified Differential Equations approach, JHEP 04 (2016) 078, [1511. 09404 ].

[7] J. R. Andersen et al., Les Houches 2017: Physics at TeV Colliders Standard Model Working Group Report, in 10th Les Houches Workshop on Physics at TeV Colliders (PhysTeV 2017) Les Houches, France, June 5-23, 2017, 2018. 1803.07977.

[8] P. Draggiotis, R. H. P. Kleiss and C. G. Papadopoulos, On the computation of multigluon amplitudes, Phys. Lett. B439 (1998) 157-164, [hep-ph/9807207].

\footnotetext{
${ }^{11}$ See also talk by Taushif Ahmed
} 
[9] A. Kanaki and C. G. Papadopoulos, HELAC: A Package to compute electroweak helicity amplitudes, Comput. Phys. Commun. 132 (2000) 306-315, [hep-ph/ 0002082$].$

[10] F. A. Berends and W. T. Giele, Recursive Calculations for Processes with $n$ Gluons, Nucl. Phys. B306 (1988) 759-808.

[11] R. Britto, F. Cachazo and B. Feng, New recursion relations for tree amplitudes of gluons, Nucl. Phys. B715 (2005) 499-522, [hep-th/ 0412308$].$

[12] R. Britto, F. Cachazo, B. Feng and E. Witten, Direct proof of tree-level recursion relation in Yang-Mills theory, Phys. Rev. Lett. 94 (2005) 181602, [hep-th/ 0501052 ].

[13] S. J. Parke and T. R. Taylor, An Amplitude for n Gluon Scattering, Phys. Rev. Lett. 56 (1986) 2459.

[14] Z. Bern, L. J. Dixon, D. C. Dunbar and D. A. Kosower, Fusing gauge theory tree amplitudes into loop amplitudes, Nucl.Phys. B435 (1995) 59-101, [hep-ph/ 9409265$].$

[15] Z. Bern, L. J. Dixon, D. C. Dunbar and D. A. Kosower, One loop n point gauge theory amplitudes, unitarity and collinear limits, Nucl.Phys. B425 (1994) 217-260, [hep-ph / 9403226 ].

[16] C. F. Berger, Z. Bern, L. J. Dixon, F. Febres Cordero, D. Forde, H. Ita et al., An Automated Implementation of On-Shell Methods for One-Loop Amplitudes, Phys. Rev. D78 (2008) 036003, [0803.4180].

[17] G. Ossola, C. G. Papadopoulos and R. Pittau, Reducing full one-loop amplitudes to scalar integrals at the integrand level, Nucl.Phys. B763 (2007) 147-169, [hep-ph/ 0609007 ].

[18] G. Ossola, C. G. Papadopoulos and R. Pittau, On the Rational Terms of the one-loop amplitudes, JHEP 0805 (2008) 004, [0 802 . 1876].

[19] SM AND NLO MULTILEG AND SM MC Working GROUPS collaboration, J. Alcaraz Maestre et al., The SM and NLO Multileg and SM MC Working Groups: Summary Report, 1203.6803.

[20] R. K. Ellis, Z. Kunszt, K. Melnikov and G. Zanderighi, One-loop calculations in quantum field theory: from Feynman diagrams to unitarity cuts, Phys.Rept. 518 (2012) 141-250, [1105. 4319 ].

[21] H. van Deurzen, G. Luisoni, P. Mastrolia, G. Ossola and Z. Zhang, Automated Computation of Scattering Amplitudes from Integrand Reduction to Monte Carlo tools, Nucl. Part. Phys. Proc. 267-269 (2015) 140-149.

[22] J. Gluza, K. Kajda and D. A. Kosower, Towards a Basis for Planar Two-Loop Integrals, Phys.Rev. D83 (2011) 045012, [1009.0472].

[23] D. A. Kosower and K. J. Larsen, Maximal Unitarity at Two Loops, Phys.Rev. D85 (2012) 045017 , [1108.1180].

[24] S. Caron-Huot and K. J. Larsen, Uniqueness of two-loop master contours, JHEP 1210 (2012) 026, [1205.0801].

[25] H. Johansson, D. A. Kosower and K. J. Larsen, Two-Loop Maximal Unitarity with External Masses, Phys. Rev. D87 (2013) 025030, [1208.1754].

[26] H. Johansson, D. A. Kosower and K. J. Larsen, An Overview of Maximal Unitarity at Two Loops, PoS LL2012 (2012) 066, [1212. 2132].

[27] H. Johansson, D. A. Kosower and K. J. Larsen, Maximal Unitarity for the Four-Mass Double Box, Phys. Rev. D89 (2014) 125010, [1308.4632]. 
[28] M. Søgaard and Y. Zhang, Multivariate Residues and Maximal Unitarity, JHEP 12 (2013) 008, $[1310.6006]$.

[29] H. Ita, Two-loop Integrand Decomposition into Master Integrals and Surface Terms, Phys. Rev. D94 (2016) 116015, [1510.05626].

[30] H. Johansson, D. A. Kosower, K. J. Larsen and M. Søgaard, Cross-Order Integral Relations from Maximal Cuts, Phys. Rev. D92 (2015) 025015, [1503.06711].

[31] P. Mastrolia, T. Peraro and A. Primo, Adaptive Integrand Decomposition in parallel and orthogonal space, JHEP 08 (2016) 164, [1605.03157].

[32] P. Mastrolia and G. Ossola, On the Integrand-Reduction Method for Two-Loop Scattering Amplitudes, JHEP 1111 (2011) 014, [1107.6041].

[33] S. Badger, H. Frellesvig and Y. Zhang, Hepta-Cuts of Two-Loop Scattering Amplitudes, JHEP 1204 (2012) 055, [1202.2019].

[34] P. Mastrolia, E. Mirabella, G. Ossola and T. Peraro, Integrand-Reduction for Two-Loop Scattering Amplitudes through Multivariate Polynomial Division, Phys. Rev. D87 (2013) 085026, [1209.4319].

[35] S. Badger, H. Frellesvig and Y. Zhang, A Two-Loop Five-Gluon Helicity Amplitude in QCD, JHEP 1312 (2013) 045, [1310.1051].

[36] C. Papadopoulos, R. Kleiss and I. Malamos, Reduction at the integrand level beyond NLO, PoS Corfu2012 (2013) 019.

[37] S. Badger, G. Mogull, A. Ochirov and D. O'Connell, A Complete Two-Loop, Five-Gluon Helicity Amplitude in Yang-Mills Theory, JHEP 10 (2015) 064, [1507.08797].

[38] K. Chetyrkin and F. Tkachov, Integration by Parts: The Algorithm to Calculate beta Functions in 4 Loops, Nucl.Phys. B192 (1981) 159-204.

[39] F. Tkachov, A Theorem on Analytical Calculability of Four Loop Renormalization Group Functions, Phys.Lett. B100 (1981) 65-68.

[40] S. Laporta, High precision calculation of multiloop Feynman integrals by difference equations, Int. J. Mod. Phys. A15 (2000) 5087-5159, [hep-ph/0102033].

[41] G. 't Hooft and M. Veltman, Scalar One Loop Integrals, Nucl.Phys. B153 (1979) 365-401.

[42] V. A. Smirnov, Analytic tools for Feynman integrals, Springer Tracts Mod. Phys. 250 (2012) 1-296.

[43] C. Bogner and S. Weinzierl, Feynman graph polynomials, Int.J.Mod.Phys. A25 (2010) 2585-2618, [1 002.3458$]$.

[44] K. Hepp, Proof of the Bogolyubov-Parasiuk theorem on renormalization, Commun. Math. Phys. 2 (1966) 301-326.

[45] M. Roth and A. Denner, High-energy approximation of one loop Feynman integrals, Nucl. Phys. B479 (1996) 495-514, [hep-ph/9605420].

[46] T. Binoth and G. Heinrich, An automatized algorithm to compute infrared divergent multiloop integrals, Nucl.Phys. B585 (2000) 741-759, [hep-ph/ 0004013$].$

[47] T. Binoth and G. Heinrich, Numerical evaluation of multiloop integrals by sector decomposition, Nucl. Phys. B680 (2004) 375-388, [hep-ph/ 0305234$].$ 
[48] C. Bogner and S. Weinzierl, Resolution of singularities for multi-loop integrals, Comput. Phys. Commun. 178 (2008) 596-610, [0 709 .4092].

[49] S. Borowka, G. Heinrich, S. P. Jones, M. Kerner, J. Schlenk and T. Zirke, SecDec-3.0: numerical evaluation of multi-scale integrals beyond one loop, Comput. Phys. Commun. 196 (2015) 470-491, [1502.06595].

[50] V. A. Smirnov, Analytical result for dimensionally regularized massless on shell double box, Phys. Lett. B460 (1999) 397-404, [hep-ph/ 9905323 ].

[51] J. B. Tausk, Nonplanar massless two loop Feynman diagrams with four on-shell legs, Phys. Lett. B469 (1999) 225-234, [hep-ph/9909506].

[52] M. Czakon, Automatized analytic continuation of Mellin-Barnes integrals, Comput. Phys. Commun. 175 (2006) 559-571, [hep-ph/ 0511200$].$

[53] A. Kotikov, Differential equations method: New technique for massive Feynman diagrams calculation, Phys.Lett. B254 (1991) 158-164.

[54] A. Kotikov, Differential equation method: The Calculation of N point Feynman diagrams, Phys.Lett. B267 (1991) 123-127.

[55] Z. Bern, L. J. Dixon and D. A. Kosower, Dimensionally regulated one loop integrals, Phys.Lett. B302 (1993) 299-308, [hep-ph/9212308].

[56] E. Remiddi, Differential equations for Feynman graph amplitudes, Nuovo Cim. A110 (1997) 1435-1452, [hep-th/9711188].

[57] T. Gehrmann and E. Remiddi, Differential equations for two loop four point functions, Nucl.Phys. B580 (2000) 485-518, [hep-ph/9912329].

[58] A. B. Goncharov, Multiple polylogarithms, cyclotomy and modular complexes, Math.Res.Lett. 5 (1998) 497-516, [1105.2076].

[59] E. Remiddi and J. Vermaseren, Harmonic polylogarithms, Int.J.Mod.Phys. A15 (2000) 725-754, [hep-ph/9905237].

[60] A. Goncharov, Multiple polylogarithms and mixed Tate motives, math/ 0103059.

[61] J. M. Henn, Multiloop integrals in dimensional regularization made simple, Phys.Rev.Lett. 110 (2013) 251601, [1304.1806].

[62] J. Ablinger, A. Behring, J. Blümlein, A. De Freitas, A. von Manteuffel and C. Schneider, Calculating Three Loop Ladder and V-Topologies for Massive Operator Matrix Elements by Computer Algebra, Comput. Phys. Commun. 202 (2016) 33-112, [1509.08324].

[63] R. N. Lee, Reducing differential equations for multiloop master integrals, JHEP 04 (2015) 108, [1411.0911].

[64] C. Meyer, Algorithmic transformation of multi-loop master integrals to a canonical basis with CANONICA, Comput. Phys. Commun. 222 (2018) 295-312, [1 705 . 06252].

[65] O. Gituliar and V. Magerya, Fuchsia: a tool for reducing differential equations for Feynman master integrals to epsilon form, Comput. Phys. Commun. 219 (2017) 329-338, [1701. 04269 ].

[66] L. Adams, C. Bogner and S. Weinzierl, The two-loop sunrise integral around four space-time dimensions and generalisations of the Clausen and Glaisher functions towards the elliptic case, J. Math. Phys. 56 (2015) 072303, [1504.03255]. 
[67] R. Bonciani, V. Del Duca, H. Frellesvig, J. M. Henn, F. Moriello and V. A. Smirnov, Two-loop planar master integrals for Higgs $\rightarrow 3$ partons with full heavy-quark mass dependence, JHEP 12 (2016) 096, [1609.06685].

[68] J. Ablinger, J. Blümlein, A. De Freitas, M. van Hoeij, E. Imamoglu, C. G. Raab et al., Iterated Elliptic and Hypergeometric Integrals for Feynman Diagrams, J. Math. Phys. 59 (2018) 062305, [1706.01299].

[69] J. L. Bourjaily, A. J. McLeod, M. Spradlin, M. von Hippel and M. Wilhelm, The Elliptic Double-Box Integral: Massless Amplitudes Beyond Polylogarithms, 1712.02785.

[70] J. Broedel, C. Duhr, F. Dulat, B. Penante and L. Tancredi, Elliptic symbol calculus: from elliptic polylogarithms to iterated integrals of Eisenstein series, 1803.10256.

[71] C. Wever and C. G. Papadopulos, in preparation, (2018).

[72] Z. Bern, L. J. Dixon and D. A. Kosower, Dimensionally regulated pentagon integrals, Nucl. Phys. B412 (1994) 751-816, [hep-ph/9306240].

[73] E. Remiddi and L. Tancredi, Differential equations and dispersion relations for Feynman amplitudes. The two-loop massive sunrise and the kite integral, Nucl. Phys. B907 (2016) 400-444, [1602.01481].

[74] C. G. Papadopoulos, Simplified differential equations approach for Master Integrals, JHEP 1407 (2014) 088, [1401.6057].

[75] J. M. Henn, K. Melnikov and V. A. Smirnov, Two-loop planar master integrals for the production of off-shell vector bosons in hadron collisions, JHEP 1405 (2014) 090, [14 02 . 70 78].

[76] C. G. Papadopoulos, D. Tommasini and C. Wever, Two-loop Master Integrals with the Simplified Differential Equations approach, JHEP 01 (2015) 072, [1409.6114].

[77] T. Gehrmann, A. von Manteuffel and L. Tancredi, The two-loop helicity amplitudes for $q \bar{q}^{\prime} \rightarrow V_{1} V_{2} \rightarrow 4$ leptons, JHEP 09 (2015) 128, [1503.04812].

[78] F. Caola, J. M. Henn, K. Melnikov and V. A. Smirnov, Non-planar master integrals for the production of two off-shell vector bosons in collisions of massless partons, JHEP 09 (2014) 043, [14 04.5590 ].

[79] C. Anastasiou, C. Duhr, F. Dulat, F. Herzog and B. Mistlberger, Higgs Boson Gluon-Fusion Production in QCD at Three Loops, Phys. Rev. Lett. 114 (2015) 212001, [1503. 06056 ]. 